\title{
Effect of CYP2C19 polymorphisms on the clinical outcome of low- dose clobazam therapy in Japanese patients with epilepsy.
}

\section{$\operatorname{AUTHOR}(\mathrm{S})$ :}

Hashi, Sachiyo; Yano, Ikuko; Shibata, Mai; Masuda, Satohiro; Kinoshita, Masako; Matsumoto, Riki; Ikeda, Akio; Takahashi, Ryosuke; Matsubara, Kazuo

\section{CITATION:}

Hashi, Sachiyo ...[et al]. Effect of CYP2C19 polymorphisms on the clinical outcome of lowdose clobazam therapy in Japanese patients with epilepsy.. European journal of clinical pharmacology 2014, 71(1): 51-58

\section{ISSUE DATE:}

2014-10-18

\section{URL:}

http://hdl.handle.net/2433/200178

\section{RIGHT:}

The final publication is available at Springer via http://dx.doi.org/10.1007/s00228-014-1773-z.; The full-text file will be made open to the public on 18 October 2015 in accordance with publisher's 'Terms and Conditions for Self-Archiving'. This is not the published version. Please cite only the published version.; この論文は出版社版でありません。引用の際 には出版社版をご確認ご利用ください。 
Effect of CYP2C19 polymorphisms on the clinical outcome of low-dose clobazam therapy in Japanese patients with epilepsy

Sachiyo Hashi $i^{\mathrm{a}}$, Ikuko Yano ${ }^{\mathrm{a}, \mathrm{b}^{*}}$, Mai Shibata ${ }^{\mathrm{a}, \mathrm{b}}$, Satohiro Masuda ${ }^{\mathrm{a}, 2}$, Masako Kinoshita ${ }^{\mathrm{c}}$, Riki Matsumoto d,1 , Akio Ikeda $^{\mathrm{d}, 1}$, Ryosuke Takahashi ${ }^{\mathrm{d}}$, Kazuo Matsubara ${ }^{\mathrm{a}}$

aDepartment of Clinical Pharmacology and Therapeutics, Kyoto University Hospital, Sakyo-ku, Kyoto 606-8507, Japan

bepartment of Clinical Pharmacy and Education, Graduate School of Pharmaceutical Sciences, Kyoto University, Sakyo-ku, Kyoto 606-8501, Japan

'Department of Neurology, Utano National Hospital, National Hospital Organization, Ukyo-ku, Kyoto 616-8255, Japan

dDepartment of Neurology, Graduate School of Medicine, Kyoto University, Sakyo-ku, Kyoto 606-8507, Japan

Corresponding author: Ikuko Yano, Ph.D.

Department of Clinical Pharmacy and Education

Graduate School of Pharmaceutical Sciences

Kyoto University

Sakyo-ku, Kyoto 606-8501, Japan 
Tel: +81-75-751-3582

Fax: +81-75-751-3205

E-mail: iyano@kuhp.kyoto-u.ac.jp

${ }^{1}$ Present Affiliation; Department of Epilepsy, Movement Disorders and Physiology, Graduate School of Medicine, Kyoto University, Sakyo-ku, Kyoto 606-8507, Japan

${ }^{2}$ Present Affiliation; Department of Pharmacy, Kyushu University Hospital. Maidashi, Higashi-ku,

Fukuoka 812-8582, Japan 


\section{Abstract}

Purpose Clobazam (CLB) is metabolized by cytochrome P450 (CYP) 3A4 to yield $N$-desmethylclobazam

( $N$-CLB), which is further inactivated by CYP2C19. The aim of this study was to retrospectively evaluate the relationship between CYP2C19 polymorphisms and the efficacy of low-dose, add-on CLB therapy in Japanese patients with epilepsy.

Methods Fifty patients were divided into three groups according to their CYP2C19 polymorphism. CLB and $N$-CLB serum concentrations, and seizure frequency before and after starting CLB were analyzed.

Results Extensive metabolizers (EMs, $\mathrm{n}=11$ ), intermediate metabolizers (IMs, $\mathrm{n}=22$ ), and poor metabolizers $(\mathrm{PMs}, \mathrm{n}=17)$ were included. Although the dose-normalized CLB serum concentrations were not significantly different, the dose-normalized $N$-CLB serum concentrations were significantly higher in PMs than in EMs or IMs. Seizure frequency was significantly decreased by the CLB therapy in PMs $(p<0.01)$, but not in EMs or IMs. CLB serum concentrations did not correlate with seizure reduction rate, but median $N$-CLB serum concentrations were significantly higher in patients with excellent seizure control ( $\geqq 90 \%$ seizure reduction) compared to those with $\geqq 50 \%$ seizure reduction or with $<50 \%$ seizure reduction (1103 ng/mL, $341 \mathrm{ng} / \mathrm{mL}$ and $570 \mathrm{ng} / \mathrm{mL}$, respectively).

Conclusions The efficacy of low-dose CLB therapy was significantly influenced by CYP2C19 polymorphisms. Ideally, CLB therapy should be started with a low dose $(2.5 \mathrm{mg} / \mathrm{d})$, and dosage increased until $N$-CLB serum concentration reaches $1100 \mathrm{ng} / \mathrm{mL}$, or until the desired effect is acquired, a recommendation that is particularly important for PMs. 
Keywords: clobazam, $N$-desmethylclobazam, CYP2C19 poor metabolizer, low-dose therapy, therapeutic drug monitoring 


\section{Introduction}

Clobazam (CLB) was approved in France in 1974 and is currently available in over 100 countries

[1]. It has been on the market since 2000 in Japan and was licensed for Lennox-Gastaut syndrome in the

United States in 2011. Other benzodiazepines such as diazepam, clonazepam, and lorazepam are 1,4-benzodiazepines, yet the efficacy of CLB, a 1,5-benzodiazepine, seems superior to that of the 1,4-benzodiazepines [2-4]. In an add-on trial, CLB administration was proven to be effective as an adjuvant therapy for epilepsy [2]. CLB has been used mainly as an add-on drug throughout the world [5-7].

CLB is metabolized primarily in the liver by cytochrome P450 (CYP) 3A4 into a pharmacologically active metabolite, $N$-desmethylclobazam ( $N$-CLB); $N$-CLB is further metabolized into an inactive metabolite by CYP2C19 [8]. CLB and N-CLB have been reported to interact with other antiepileptic drugs [9-14]. CLB concentrations are decreased by the presence of carbamazepine, phenytoin, or phenobarbital, which are characterized as CYP inducers, inducing CYP3A4 as well [9-11]. $N$-CLB concentrations were reported to increase when CLB was concurrently administered with carbamazepine or phenytoin, which presumably occurs because the concurrently administered drugs induce the metabolism of CLB to $N$-CLB [11-13]. In contrast, zonisamide and topiramate are reported to be CYP2C19 inhibitors $[14,15]$ and can increase $N$-CLB concentrations.

Not only the co-administrated drugs but also polymorphic recessive mutations at the CYP2C19 gene, which cause the poor metabolizer (PM) phenotype [16], are known to affect the metabolism of CLB. The $N$-CLB/CLB concentration ratio was dependent on the number of mutated alleles of CYP2C19 (gene 
dose effect), and this ratio may be a valuable parameter to screen for patients at risk for side effects $[17,18]$. The frequency of CYP2C19 PM varies across races, and is higher in Asian populations (18-23\%), including Japanese, than in Caucasians (2-5\%) [16,19]. Two defective CYP2C19 alleles (CYP2C19*2 and CYP2C19*3) have been found to account for more than 99\% of Asian PM alleles, and allele frequencies for CYP2C19*2 and CYP2C19*3 were 0.31 and 0.10 , respectively, in the Japanese population [20].

CLB is usually administered at a daily dose of $10-40 \mathrm{mg}$. However, we previously reported that the low-dose $(2.5 \mathrm{mg} / \mathrm{d})$, add-on CLB therapy is effective in some adult patients with refractory epilepsy and that its degree of effectiveness is correlated with serum concentrations of $N$-CLB [21]. However, information on the effect of CYP2C19 polymorphisms on the clinical efficacy and therapeutic range of $\mathrm{N}$-CLB is limited. The objective of this study was to retrospectively analyze the effect of CYP2C19 genotypes on the pharmacokinetics of CLB and $N$-CLB, as well as the clinical efficacy in the Japanese adult patients with epilepsy. We clarified the relationship between CYP2C19 polymorphisms and clinical efficacy in the low-dose (initial dose, $2.5 \mathrm{mg} / \mathrm{d}$ ), add-on CLB therapy, and also defined a clinically effective serum concentration range for $N$-CLB.

\section{Materials and Methods}

\section{Subject and study design}

This retrospective study included 50 adult patients (24 men and 26 women; age range, 18-72 years) with epilepsy who had been treated at the epilepsy clinics of the Department of Neurology, Kyoto 
University Hospital from July 2006 to September 2011. These patients had been receiving the same dosage of CLB regularly for at least one month. This study was conducted according to the Declaration of Helsinki and its amendments, and was approved by the Kyoto University Graduate School of Medicine, Faculty of Medicine, and the Kyoto University Hospital Ethics Committee. All patients provided written informed consent before undergoing the genotyping test.

The steady state dose was individually decided as follows. As an add-on therapy, CLB was started in patients with $2.5 \mathrm{mg} / \mathrm{d}$ at bedtime, with both efficacy and tolerability observed at least four weeks later. In this study, the tolerability is defined as the state that patients could keep the same dose of medication with tolerable adverse reactions, such as slight sleepiness that their quality of life was not significantly impaired. Until the patients had improved seizure control with tolerable adverse reactions, the dose was increased by $2.5 \mathrm{mg} / \mathrm{d}$ every at least four weeks. Once better seizure control was achieved, the same dose was retained as the steady state dose. If intolerable side effects such as sleepiness, excessive drooling, or drowsiness occurred, the dose was decreased by $2.5 \mathrm{mg} / \mathrm{d}$ or finally stopped, depending on the clinical state. Throughout those periods, other antiepileptic drugs were maintained at the same dose.

\section{Pharmacokinetics}

Serum drug concentrations in 50 samples were retrospectively analyzed. These blood samples were collected during the outpatient visit in the morning after steady state for the optimal dose had been achieved. Concentrations of CLB and N-CLB were measured with high-performance liquid chromatography (HPLC) with UV detection by a validated external commission company (SRL Inc., 
Tokyo, Japan). The lower limit of quantification was $10 \mathrm{ng} / \mathrm{mL}$ for both drugs. The intra- and inter-day variation of the analysis was 3.0\% and 6.0\%, respectively. Samples with concentrations of CLB under the lower limit of detection $(10 \mathrm{ng} / \mathrm{mL})$ because of low dosage were included during in the analysis and replaced with the value of $5 \mathrm{ng} / \mathrm{mL}(\mathrm{n}=5)$ [22]. Two patients who did not have CLB or $N$-CLB concentration datum, but were evaluated the clinical efficacy were included in the study. We also collected information on concurrent therapy with antiepileptic drugs, carbamazepine, phenytoin, valproic acid, zonisamide, phenobarbital, clonazepam, gabapentin, topiramate, lamotrigine, and levetiracetam, from the patients' medical records. All patients were administering 1-5 other antiepileptic drugs, in various combinations. Patients were classified as those who received CYP inducers (phenytoin, carbamazepine, or phenobarbital) and those who received CYP2C19 inhibitors (zonisamide or topiramate) $[14,15,23]$. Each patient was not receiving any drugs except antiepileptics which potentially affect the pharmacokinetics of CLB, such as CYP3A4 inhibitors or CYP2C19 inhibitors.

\section{Genotyping}

Genomic DNA was isolated from whole blood samples, and the polymerase chain reaction restriction fragment length polymorphism method was used to analyze CYP2C19*2 and CYP2C19*3 mutations [24,25]. The patients were divided into three groups according to their CYP2C19 polymorphisms: homozygous extensive metabolizers (EMs) $(* 1 / * 1)$, intermediate metabolizers (IMs) $(* 1 / * 2$ or $* 1 / * 3)$, and poor metabolizers (PMs) $(* 2 / * 2, * 3 / * 3$ or $* 2 / * 3)$. The characteristics of patients distributed into these three groups were compared. 


\section{Evaluation of epilepsy}

In accordance with the epilepsy classification in 2010 as much as possible to apply [26], 33 cases were classified as partial epilepsy with multiple etiologies (being equivalent to 24 cases as temporal lobe epilepsy and 9 as extra-temporal lobe epilepsy in 1989 classification [27]), 17 patients as with epilepsy with generalized tonic-clonic seizures alone or generalized epilepsy with multiple etiologies (being equivalent to symptomatic generalized epilepsy or idiopathic generalized epilepsy in 1989 classification [27]). With regard to seizure classification [26], 9 and two patients had two or three types of seizures, respectively, and the others had one type of seizure: 43 patients had focal seizures (being equivalent to 27 had complex partial seizures, and 16 had simple partial seizures in 1981 classification [28]), and 20 patients had generalized tonic-clonic seizures.

Baseline seizure frequency and seizure frequency during CLB therapy was determined by counting the number of seizures in the three months immediately prior to administration of CLB, and after reaching the optimal dose of CLB, respectively. Patients whose seizure frequency was more than one per month during the baseline period, despite receiving the maximum tolerable dose of appropriate antiepileptic drugs in various combinations, were judged as having intractable seizures with the rest characterized as having tractable seizures. Clinical efficacies were evaluated based upon seizure control. The patients whose seizure frequency was reduced by less than $50 \%$, or whose CLB administration was withdrawn were classified into the “<50\% seizure reduction” group. The patients whose seizure frequency was reduced by more than $50 \%$, but less than $90 \%$ were classified into the “ $\geqq 50 \%$ seizure 
reduction” group. The patients whose seizure frequencies were reduced by more than $90 \%$, were classified into the " $\geqq 90 \%$ seizure reduction" group. The physician-in-charge, who was blinded to the results of CYP2C19 polymorphisms, evaluated clinical outcomes in each patient. Three patients (one from each group) who suffered from psychogenic seizures were excluded from the analysis of seizure frequency at the baseline and clinical efficacy.

\section{Statistical analysis}

Statistical analyses were performed using Prism6 software (GraphPad Software, San Diego, CA, USA). Differences between the three groups were analyzed using the Kruskal-Wallis test, followed by Dunn's multiple comparison test. Frequencies of observation among the three CYP2C19 phenotypes were analyzed using the chi-square test. The CYP2C19 genotype frequency of the study patients was analyzed with the Hardy-Weinberg principle using the chi-square test. Changes in seizure frequency before and after initiation of CLB therapy were analyzed by the paired t-test. A $p$ value less than 0.05 was considered to be statistically significant.

\section{Results}

Fifty patients were divided into three groups according to their CYP2C19 phenotype: EMs ( $\mathrm{n}=$ 11), IMs ( $\mathrm{n}=22)$, and PMs $(\mathrm{n}=17)$ (Table 1). There were no significant differences between the three groups in terms of gender, age, epilepsy classification, seizure type, seizure frequency at baseline, or following co-administration of a CYP inducer or CYP2C19 inhibitor. The body weight and CLB dose 
were significantly different between the three phenotypes, and the CLB dose per body weight in PMs was less than half in EMs, although this difference did not reach statistical significance. The genotype frequency of CYP2C19 in the 50 patients was inconsistent with the Hardy-Weinberg equilibrium based on the allele frequency of CYP2C19*2 and CYP2C19*3 [20] $(p<0.01)$.

The median time from the start of CLB administration to the measurement of serum concentration was 12 months (1-69 months), 17 months (1-75 months) and 5 months (1-235 months) in EMs, IMs and PMs, respectively. The serum concentrations of CLB and N-CLB were compared between the three groups (Table 1). $N$-CLB concentrations were higher in PMs (median, $1071 \mathrm{ng} / \mathrm{mL}$ ) than in IMs (median, $632 \mathrm{ng} / \mathrm{mL}$ ) or EMs (median, $290 \mathrm{ng} / \mathrm{mL}$ ), although no significant differences in CLB concentration were observed between the three groups. To adjust the effect of dose differences among the groups, the ratio of serum concentration to CLB dose (C/D ratio) for CLB and N-CLB, and the serum concentration ratio of $N$-CLB to CLB ( $N$-CLB/CLB ratio) were calculated for the three groups (Fig. 1). The $N$-CLB C/D ratio was significantly higher in PMs (median, $16300(\mathrm{ng} / \mathrm{mL}) /(\mathrm{mg} / \mathrm{kg} / \mathrm{d})$ ) than in EMs (median, 1760 $(\mathrm{ng} / \mathrm{mL}) /(\mathrm{mg} / \mathrm{kg} / \mathrm{d})$ ) or IMs (median, $5640(\mathrm{ng} / \mathrm{mL}) /(\mathrm{mg} / \mathrm{kg} / \mathrm{d}))$, while the CLB C/D ratio did not significantly differ between the three groups. The $N$-CLB/CLB ratio was also significantly higher in PMs (median, 63.5) than in EMs (median, 5.4) or IMs (median, 15.3).

To evaluate the effect of co-administered drugs on the pharmacokinetics of CLB, the patients were divided into 4 groups: patients who were not administered CYP inducers nor CYP2C19 inhibitors, patients administered CYP2C19 inhibitors without CYP inducers, patients administered CYP inducers without CYP2C19 inhibitors, and patients administered both CYP inducers and CYP2C19 inhibitors. The 
CLB C/D ratio was significantly lower in the group administered CYP inducers compared to the group that was administered neither CYP inducers nor CYP2C19 inhibitors, or to the group administered only CYP2C19 inhibitors. There were no significant differences in the $N$-CLB C/D ratio between the 4 groups (Fig. 2), while within each group, the N-CLB C/D ratio in PMs tended to be higher than that in EMs or IMs.

To evaluate the clinical effect of CLB, changes in seizure frequency before and after starting CLB therapy were analyzed for the three CYP2C19 phenotypes (Fig. 3). Seizure frequency was decreased in all PMs except one patient. There were no significant changes in seizure frequency for either EMs or IMs.

The clinical effect was evaluated by determining the seizure-reduction rate in the three months prior to and following CLB add-on therapy (Fig. 4). Patients were divided into $<50 \%$-reduction, $\geqq$ $50 \%$-reduction, and $\geqq 90 \%$-reduction groups, and concentrations of CLB and $N$-CLB were compared between the three groups. $N$-CLB concentration was significantly higher in the $\geqq 90 \%$-reduction group (median value: $1103 \mathrm{ng} / \mathrm{mL}$ ) than in the $<50 \%$ or the $\geqq 50 \%$-reduction groups (median value: $570 \mathrm{ng} / \mathrm{mL}$ and $341 \mathrm{ng} / \mathrm{mL}$, respectively). There were no significant differences in CLB concentration between these groups (median value: 57,30 , and $35 \mathrm{ng} / \mathrm{mL}$ for $<50 \%$, $\geqq 50 \%$, and $\geqq 90 \%$-reduction group, respectively).

\section{Discussion}

CLB is metabolized mainly by CYP3A4, and its active metabolite $N$-CLB is further inactivated by CYP2C19. It has been reported that the antiepileptic potency of $N$-CLB is $40 \%$ of CLB [29], and that 
serum concentrations of $N$-CLB are affected by CYP2C19 polymorphisms $[17,18,30]$. The effect of CYP2C19 polymorphisms on the clinical efficacy of CLB was recently described in one report of pediatric cases [30], but was not reported in adult patients. Moreover, information concerning the serum concentration range for clinically effective CLB or N-CLB is unknown. In this report, we analyzed the relationship between CYP2C19 polymorphisms and clinical efficacy of CLB in adult epileptic patients, and clarified the clinically therapeutic window of $N$-CLB. In our study, CLB was initiated at a low dose, since Kinoshita et al. [21] had observed that low-dose CLB therapy was effective in some patients, and was associated with fewer adverse reactions. This strategy differed from strategies used in previous reports investigating CLB therapy $[17,18,30]$.

Although the dose of CLB tended to be lower in PMs compared to that in EMs or IMs, the serum concentration of $N$-CLB and the seizure reduction rates were significantly higher in PMs (Table 1, Fig. 3). Indeed, the $N$-CLB C/D ratio and $N$-CLB/CLB ratio were significantly higher in PMs than in EMs or IMs (Fig. 1), while the CLB C/D ratio was not significantly different between the three groups. The clinical background (i.e. the epilepsy classification, seizure type, and the baseline seizure frequency) was not significantly different between the three groups. As shown in Fig. 2, concomitant drug administration did not significantly affect $N$-CLB concentrations, although the CYP inducers such as phenytoin, carbamazepine, or phenobarbital significantly decreased CLB concentrations. These findings suggest that CYP2C19 polymorphisms strongly affect $N$-CLB pharmacokinetics, resulting in differences in the clinical effectiveness of CLB. In addition, the serum concentration of $N$-CLB, but not CLB, was related to its therapeutic effect, evidenced by the fact that patients with excellent seizure control had significantly 
higher $N$-CLB serum concentrations (Fig. 4).

A previous report suggested that the serum concentrations of CLB or $N$-CLB did not correlate with clinical efficacy [31]. Neels et al. suggested that the therapeutic range of CLB was $100-400 \mathrm{ng} / \mathrm{mL}$, and that the serum concentrations of $N$-CLB were 8 times higher than serum concentrations of CLB [32]. In another report, the serum concentrations of CLB and $N$-CLB were in the order of 30-300 ng/mL and 300 $-3000 \mathrm{ng} / \mathrm{mL}$, respectively, in patients treated with therapeutic doses of CLB, although no clear relationships were indicated between efficacy and serum concentrations of CLB or N-CLB [33]. In the present study, it is noteworthy that the median serum concentration of $N$-CLB in patients with $\geqq 90 \%$ seizure reduction was $1103 \mathrm{ng} / \mathrm{mL}$, with this value being significantly higher than concentrations in patients with $<50 \%$ seizure reduction $(570 \mathrm{ng} / \mathrm{mL}$ ) and $\geqq 50 \%$ seizure reduction (341 ng/mL) groups, as shown in Fig. 4. On the other hand, the median CLB concentrations were approximately $30-57 \mathrm{ng} / \mathrm{mL}$ regardless of the seizure reduction rate. Therefore, we considered that the therapeutic effect of CLB most likely depends on $\mathrm{N}$-CLB serum concentration, with the therapeutic concentration of $\mathrm{N}$-CLB being approximately $1100 \mathrm{ng} / \mathrm{mL}$.

Kinoshita et al. [21] also reported that when CLB was started with a daily dose of $2.5 \mathrm{mg}$, and the patients whose serum $N$-CLB concentrations were higher (mean \pm SD, $738 \pm 192 \mathrm{ng} / \mathrm{mL}$ ) experienced better seizure control with fewer adverse reactions. This value is much lower than the serum concentrations of $N$-CLB in PMs that have been reported for doses of CLB used in previous studies. For example, Seo et al. [30] reported $N$-CLB serum concentrations of $7700 \pm 6040,2140 \pm 1690$, and $920 \pm 610 \mathrm{ng} / \mathrm{mL}$ (mean \pm SD) in PMs, IMs, and EMs, respectively, and Kosaki et al. [18] reported 
serum concentrations of $4806 \pm 2575 \mathrm{ng} / \mathrm{mL}$ (mean $\pm \mathrm{SD}$ ) for $N$-CLB in PMs. Serum concentrations of CLB in our study (approximately 30-57 ng/mL) were also lower than concentrations published in the previous reports. Seo et al. [30] reported CLB serum concentrations of $140-210 \mathrm{ng} / \mathrm{mL}$ for each CYP2C19 phenotype, and Kosaki et al. [18] reported 130-219 ng/mL for each CYP2C19 phenotype. We suppose that these differences are derived from our strategy of low-dose CLB therapy; initiating with a low-dose of CLB (2.5mg/d) and acquiring good clinical effects with lower dose of CLB compared to conventional CLB therapy. In fact, the stable median CLB dose in our study was $5 \mathrm{mg} / \mathrm{d}$, which was lower than in previous reports. Adverse reactions are probably reduced when lower doses of CLB are used, because higher serum concentrations of CLB or N-CLB have been supposed to be related to adverse reactions. This is one of the merits of low-dose CLB therapy.

In this study, the frequency of occurrence of the phenotype for CYP2C19 was inconsistent with the Hardy-Weinberg equilibrium (Table 1), and the proportion of PMs was relatively high (34\%), compared to the reported frequency in Asian populations (18-23\%) [16]. This is possibly because, in our retrospective study, EMs tended to drop out more frequently than patients in other groups. Although the usual recommended starting dose of CLB is $10 \mathrm{mg} / \mathrm{d}$, the starting dose of CLB set at $2.5 \mathrm{mg} / \mathrm{d}$ in our hospital resulted in inadequate serum concentrations of N-CLB in the CYP2C19 EMs. Therefore, the dosage was gradually increased. The increased CLB concentrations may have caused adverse reactions that necessitated withdrawal of CLB therapy. Although this study did not investigate the relationship between serum concentrations of CLB or $N$-CLB and side effects, this relationship remains to be clarified in a future study. In addition, the limitation of this study is the retrospective, uncontrolled design of the 
study. The prospective, controlled design of the study including a large number of patients with similar kind of epilepsies is needed in the future.

In conclusion, the efficacy of low-dose, add-on CLB therapy is significantly influenced by CYP2C19 polymorphisms. From a practical standpoint, it is better to start CLB at a low dose (2.5 mg/d) in PMs and to increase the dose aiming to achieve an $N$-CLB concentration of $1100 \mathrm{ng} / \mathrm{mL}$, until the desired effect is acquired. Such individualized therapy, guided by an analysis of CYP2C19 polymorphisms is warranted, because a good clinical effect without adverse reactions of CLB therapy would be expected in PMs.

\section{Acknowledgments}

This work was partially supported by a Grant-in-Aid for Scientific Research from the Ministry of Education, Culture, Sports, Science and Technology of Japan.

\section{Conflicts of interest}

None of the authors has any conflict of interest related to this study. 


\section{References}

1. Ng YT, Collins SD (2007) Clobazam. Neurotherapeutics 4:138-144

2. Shorvon SD (1995) Benzodiazepines: Clobazam. In: Levy RH, Mattson RH, Meldrum BS (eds) Antiepileptic drugs, 4th edn. Raven Press Ltd, New York, pp 763-777

3. Dichter MA, Brodie MJ (1996) New antiepileptic drugs. N Engl J Med 334:1583-1590

4. de Leon J, Spina E, Diaz FJ (2013) Clobazam therapeutic drug monitoring: a comprehensive review of the literature with proposals to improve future studies. Ther Drug Monit 35: 30-47

5. Canadian Clobazam Cooperative Group (1991) Clobazam in treatment of refractory epilepsy: the Canadian experience. A retrospective study. Epilepsia 32:407-416

6. Robertson MM (1986) Current status of the 1,4- and 1,5-benzodiazepines in the treatment of epilepsy: the place of clobazam. Epilepsia 27:27-41

7. Schmidt,D, Rohde M, Wolf P, Roeder-Wanner U (1986) Clobazam for refractory focal epilepsy. A controlled trial. Arch Neurol 43:824-826

8. Giraud C, Tran A, Rey E, Vincent J, Treluyer JM, Pons G (2004) In vitro characterization of clobazam metabolism by recombinant cytochrome P450 enzymes: importance of CYP2C19. Drug Metab Dispos 32:1279-1286

9. Levy RH, Lane, EA Guyot M, Brachet-Liermain A, Cenraud B, Loiseau P (1983) Analysis of parent drug-metabolic relationship in the presence of an inducer. Application to the carbamazepine-clobazam interaction in normal man. Drug Metab Dispos 11:286-292

10. Jawad S, Richens A, Oxley J (1984) Single dose pharmacokinetic study of clobazam in normal 
volunteers and epileptic patients. Br J Clin Pharmacol 18:873-877

11. Sennoue S, Mesdjian E, Bonneton J, Genton P, Dravet C, Roger J (1992) Interactions between clobazam and standard antiepileptic drugs in patients with epilepsy. Ther Drug Monit 14:269-274

12. Theis JG, Koren G, Daneman R, Sherwin AL, Menzano E, Cortez M, Hwang P (1997) Interactions of clobazam with conventional antiepileptics in children. J Child Neurol 12:208-213

13. Hachad H, Ragueneau-Majlessi I, Levy RH (2002) New antiepileptic drugs: review on drug interactions. Ther Drug Monit 24:91-103

14. Benedetti MS (2000) Enzyme induction and inhibition by new antiepileptic drugs: a review of human studies. Fundam Clin Pharmacol 14:301-319

15. Odani A, Hashimoto Y, Takayanagi K, Otsuki Y, Koue T, Takano M, Yasuhara M, Hattori H, Frusho K, Inui K (1996) Population pharmacokinetics of phenytoin in Japanese patients with epilepsy: Analysis with a dose-dependent clearance model. Biol Pharm Bull 19:444-448

16. de Morais SM, Wilkinson GR, Blaisdell J, Meyer UA, Nakamura K, Goldstein JA (1994) Identification of a new genetic defect responsible for the polymorphism of (S)-mephenytoin metabolism in Japanese. Mol Pharmacol 46:594-598

17. Contin M, Sangiorgi S, Riva R, Parmeggiani A, Albani F, Baruzzi A (2002) Evidence of polymorphic CYP2C19 involvement in the human metabolism of N-desmethylclobazam. Ther Drug Monit 24:737-741

18. Kosaki K, Tamura K, Sato R, Samejima H, Tanigawara Y, Tahakashi T (2004) A major influence of CYP2C19 genotype on the steady-state concentration of N-desmethylclobazam. Brain Dev 6:530- 
19. Goldstein JA, Faletto MB, Romkes-Sparks M, Sullivan T, Kitareewan S, Raucy JL, Lasker JM, Ghanayem BI (1994) Evidence that CYP2C19 is the major (S)-mephenytoin 4'-hydroxylase in humans. Biochemistry 33:1743-1752

20. Inoue K, Yamazaki H, Imiya K, Akasaka S, Guengerich FP, Shimada T (1997) Relationship between CYP2C9 and 2C19 genotypes and tolbutamide methyl hydroxylation and S-mephenytoin 4'-hydroxylation activities in livers of Japanese and Caucasian populations. Pharmacogenetics 7:103-113

21. Kinoshita M, Ikeda A, Begum T, Terada K, Shibasaki H (2007) Efficacy of low-dose, add-on therapy of clobazam (CLB) is produced by its major metabolite, N-desmethyl-CLB. J Neurol Sci 263:44-48

22. Beal SL (2001) Ways to fit a PK model with some data below the quantification limit. J Pharmacokinet Pharmacodyn 28:481-504

23. Anderson GD (1998) A mechanistic approach to antiepileptic drug interactions. Ann Pharmacother $32: 554-563$

24. Hosohata K, Masuda S, Katsura T, Takada Y, Kaido T, Ogura Y, Oike F, Egawa H, Uemoto S, Inui K (2009) Impact of intestinal CYP2C19 genotypes on the interaction between tacrolimus and omeprazole, but not lansoprazole, in adult living-donor liver transplant patients. Drug Metab Dispos 37:821-826

25. van Schaik RH, van der Heiden IP, van den Anker JN, Lindemans J (2002) CYP3A5 variant allele 
frequencies in Dutch Caucasians. Clin Chem 48:1668-1671

26. Berg AT, Berkovic SF, Brodie MJ, Buchhalter J, Cross JH, van Emde Boas W, Engel J, French J, Glauser TA, Mathern GW, Moshé SL, Nordli D, Plouin P, Scheffer IE (2010) Revised terminology and concepts for organization of seizures and epilepsies: report of the ILAE Commission on Classification and Terminology, 2005-2009. Epilepsia 51:676-685

27. Commission on Classification and Terminology of the International League Against Epilepsy (1989) Proposal for revised classification of epilepsies and epileptic syndromes. Epilepsia 30:389-399

28. Commission on Classification and Terminology of the International League Against Epilepsy (1981) Proposal for revised clinical and electroencephalographic classification of epileptic seizures. Epilepsia 22:489-501

29. Fielding S, Hoffmann I (1979) Pharmacology of anti-anxiety drugs with special reference to clobazam. Br J Clin Pharmacol 7:7-15

30. Seo T, Nagata R, Ishitsu T, Murata T, Takaishi C, Hori M, Nakagawa K (2008) Impact of CYP2C19 polymorphisms on the efficacy of clobazam therapy. Pharmacogenomics 9:527-537

31. Allen JW, Oxley J, Robertson MM, Trimble MR, Richens A, Jawad SS (1983) Clobazam as adjunctive treatment in refractory epilepsy. Br Med J 286:1246-1247

32. Neels HM, Sierens AC, Naelaerts K, Scharpé SL, Hatfield GM, Lambert WE (2004) Therapeutic drug monitoring of old and newer anti-epileptic drugs. Clin Chem Lab Med 42:1228-1255

33. Patsalos PN, Berry DJ, Bourgeois BF, Cloyd JC, Glauser TA, Johannessen SI, Leppik IE, Tomson T, Perucca E (2008) Antiepileptic drugs--best practice guidelines for therapeutic drug monitoring: a 
position paper by the subcommission on therapeutic drug monitoring, ILAE Commission on

Therapeutic Strategies. Epilepsia 49:1239-1276 


\section{Figure Legends}

Fig. 1. Effect of CYP2C19 polymorphisms on (a) the serum clobazam concentration/dose (CLB C/D)

ratio, (b) the serum $N$-desmethylclobazam concentration/dose ( $N$-CLB C/D) ratio, and (c) $N$-desmethylclobazam serum concentration/clobazam serum concentration ( $N$-CLB/CLB) ratio. Each symbol represents an individual patient, and each bar shows the median. Patients were divided into three groups: EMs $(* 1 / * 1)$, IMs $(* 1 / * 2$ or $* 1 / * 3)$, and PMs $(* 2 / * 2, * 3 / * 3$ or $* 2 / * 3)$. P-values were obtained using the Kruskal-Wallis test, followed by Dunn’s multiple comparison test.

Fig. 2. Effect of co-administered drugs on serum concentrations of (a) CLB and (b) N-CLB. CYP inducers are phenytoin, carbamazepine, or phenobarbital. CYP2C19 inhibitors are zonisamide or topiramate. Each symbol represents an individual patient, and each bar shows the median. Closed circles indicate CYP2C19 PM, and open circles indicate CYP2C19 EM or IM. P-values were obtained using the Kruskal-Wallis test, followed by Dunn’s multiple comparison test.

Fig. 3. Comparisons of seizure frequency before and after initiation of CLB therapy according to the CYP2C19 polymorphisms. $P$-values were obtained using the paired t- test.

Fig. 4. Relationships between the seizure reduction rate and the serum concentrations of (a) CLB and (b)

$\mathrm{N}$-CLB. Each symbol represents an individual patient, and each bar shows the median. The concentrations of CLB and N-CLB were plotted with a logarithm scale. Closed circles indicate CYP2C19 
PM, and open circles indicate CYP2C19 EM or IM. P-values were obtained using the Kruskal-Wallis test, followed by Dunn’s multiple comparison test. 
Table 1. Characteristics of patients included in the study

\begin{tabular}{|c|c|c|c|c|}
\hline Characteristic & EMs & IMs & PMs & $p$-value \\
\hline Number of patients & $11(22 \%)$ & $22(44 \%)$ & 17 (34\%) & $<0.01^{\mathrm{a}}$ \\
\hline Gender (M/F) & $5 / 6$ & $13 / 9$ & $6 / 11$ & $0.331^{\mathrm{b}}$ \\
\hline Age (year) & $38(18-51)$ & $39(19-72)$ & $34(20-61)$ & $0.242^{c}$ \\
\hline Body weight (kg) & $59.0(42.5-78.9)$ & $56.3(42.5-103.0)$ & $50.0(41.0-70.0)$ & $0.030^{c}$ \\
\hline CLB dose (mg/d) & $10(2.5-22.5)$ & $5(2.5-22)$ & $5(1.25-15)$ & $0.014^{c}$ \\
\hline $\begin{array}{l}\text { CLB dose/body weight } \\
(\mathrm{mg} / \mathrm{kg} / \mathrm{d})\end{array}$ & $0.18(0.04-0.47)$ & $0.10(0.04-0.42)$ & $0.07(0.03-0.34)$ & $0.093^{c}$ \\
\hline CLB conc. (ng/mL) & $51(5-160)$ & $36(5-339)$ & $23(5-278)$ & $0.137^{c}$ \\
\hline$N$-CLB conc. (ng/mL) & $290(72-1629)$ & $632(85-3629)$ & $1071(292-6274)$ & $0.013^{c}$ \\
\hline \multicolumn{5}{|l|}{ Epilepsy classification: } \\
\hline Temporal lobe epilepsy & 5 & 11 & 8 & $0.912^{\mathrm{b}}$ \\
\hline $\begin{array}{l}\text { Extra-temporal lobe } \\
\text { epilepsy }\end{array}$ & 3 & 3 & 3 & \\
\hline $\begin{array}{l}\text { Symptomatic or } \\
\text { idiopathic generalized } \\
\text { epilepsy }\end{array}$ & 3 & 8 & 6 & \\
\hline \multicolumn{5}{|l|}{ Seizure type: } \\
\hline $\begin{array}{l}\text { Complex partial } \\
\text { seizures }\end{array}$ & 5 & 16 & 6 & $0.334^{\mathrm{b}}$ \\
\hline Simple partial seizures & 4 & 5 & 7 & \\
\hline $\begin{array}{l}\text { Generalized } \\
\text { tonic-clonic seizures }\end{array}$ & 6 & 7 & 7 & \\
\hline $\begin{array}{l}\text { Seizure frequency at } \\
\text { baseline: }\end{array}$ & & & & \\
\hline Intractable & 9 & 14 & 14 & $0.190^{\mathrm{b}}$ \\
\hline Tractable & 1 & 7 & 2 & \\
\hline $\begin{array}{l}\text { Co-administered } \\
\text { AEDs: }\end{array}$ & & & & \\
\hline CYP inducer ${ }^{\mathrm{d}}(+/-)$ & $6 / 5$ & $16 / 6$ & $13 / 4$ & $0.434^{\mathrm{b}}$ \\
\hline $\begin{array}{l}\text { CYP2C19 } \\
\text { inhibitor }^{\mathrm{e}}(+/-)\end{array}$ & $2 / 9$ & $6 / 16$ & $7 / 10$ & $0.402^{\mathrm{b}}$ \\
\hline
\end{tabular}

Data are shown as median (range) or number of patients

a: Hardy-Weinberg equilibrium test for CYP2C19 phenotype $\quad$ b: $p$-value by Chi-square test

c: $p$-value by Kruskal-Wallis test $\mathrm{d}$ : phenytoin, carbamazepine, or phenobarbital

e: zonisamide or topiramate 


\section{Fig.1}

a)

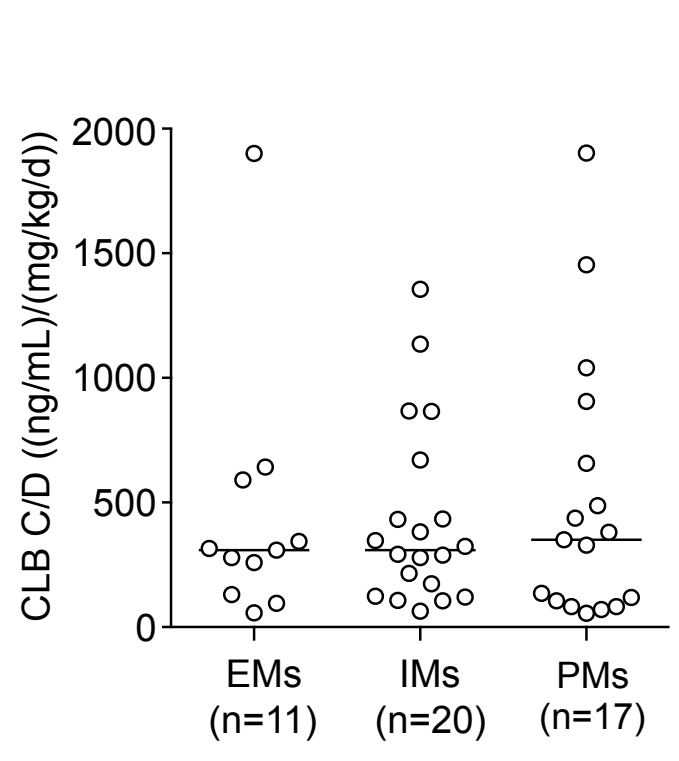

b)

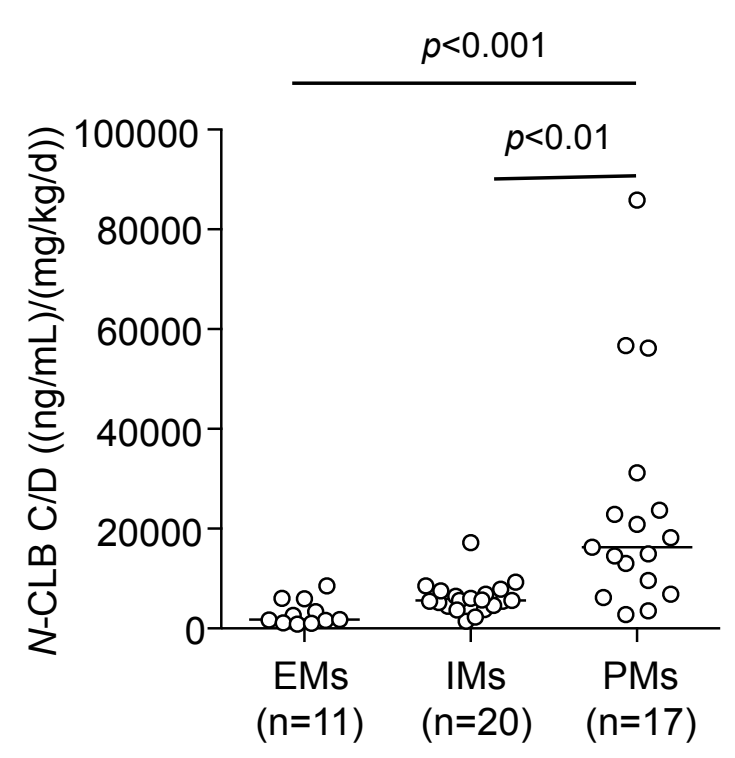

c)

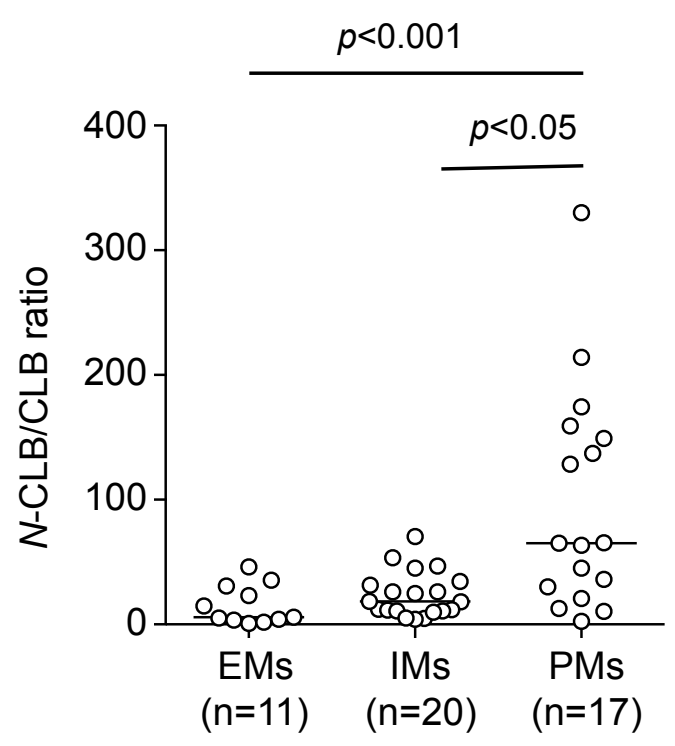


Fig. 2

a)

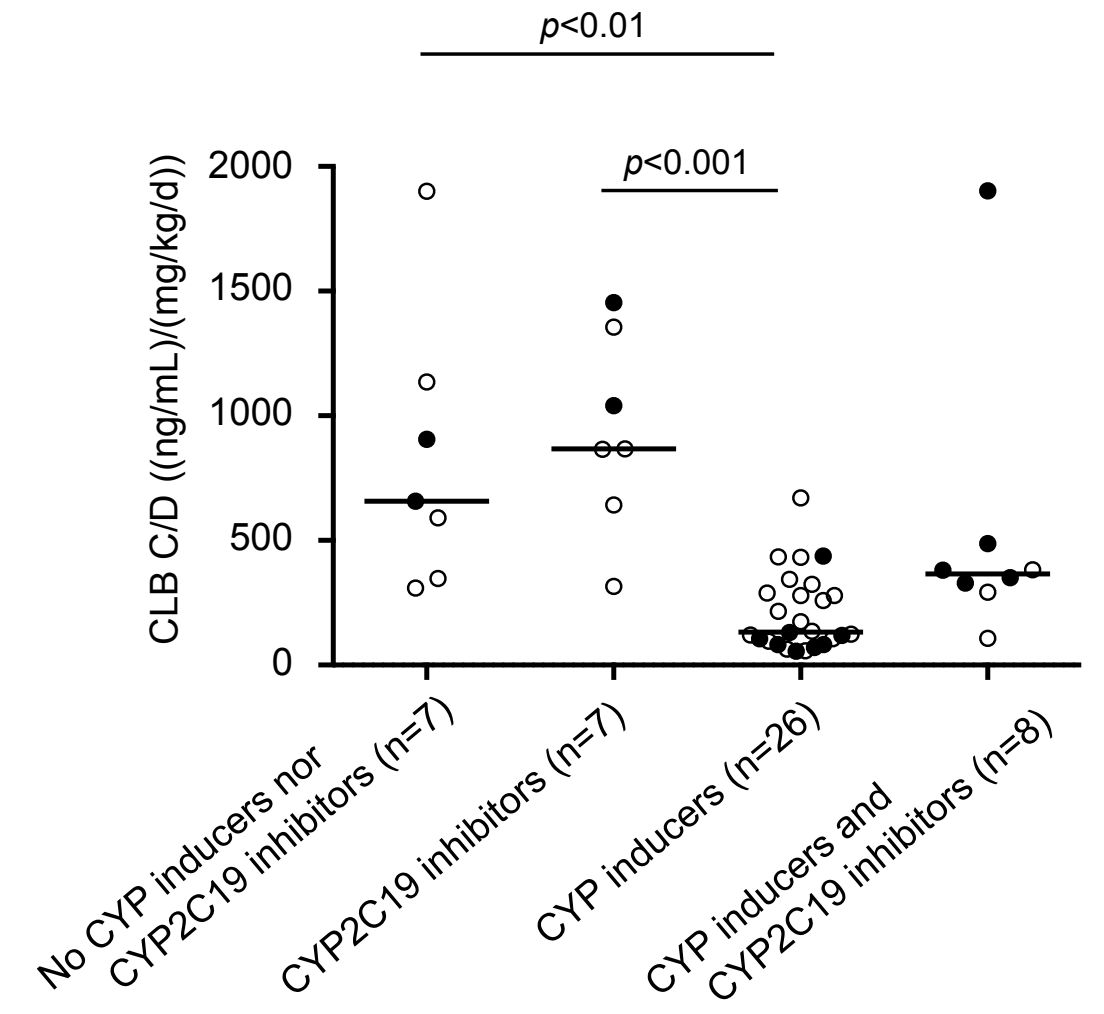

b)

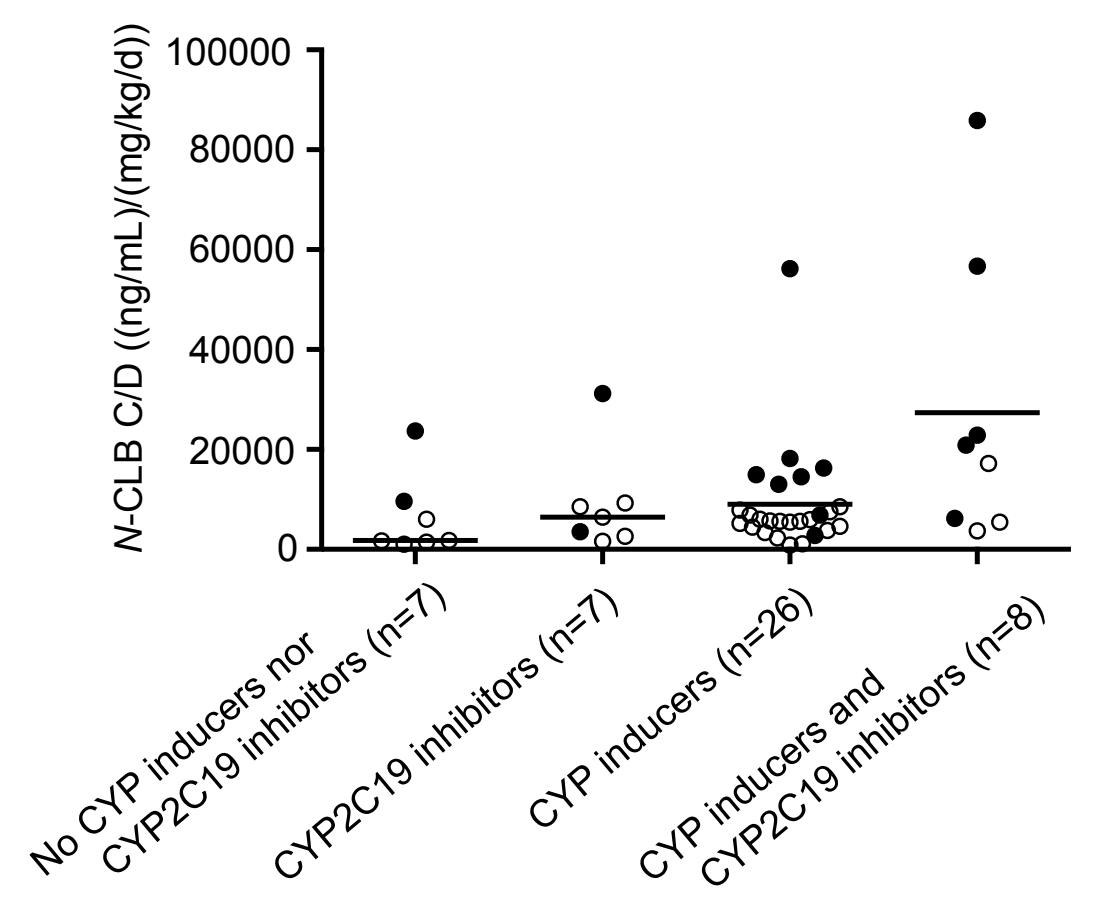




\section{Fig.3}

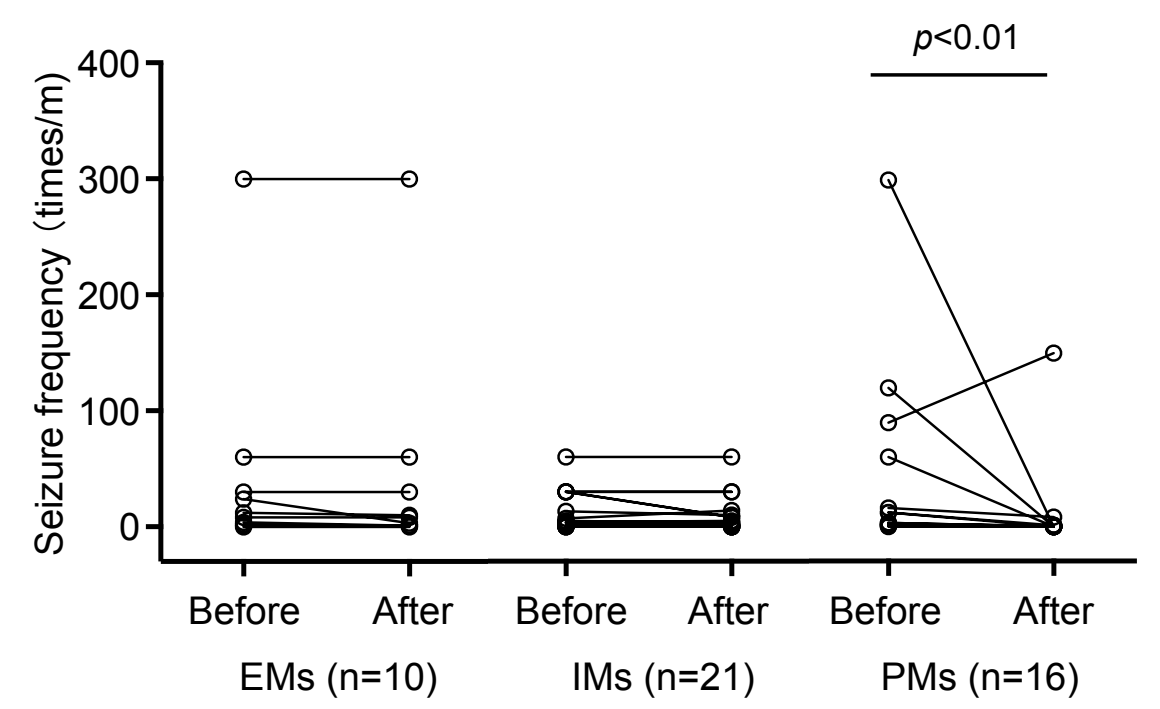


Fig.4

a)

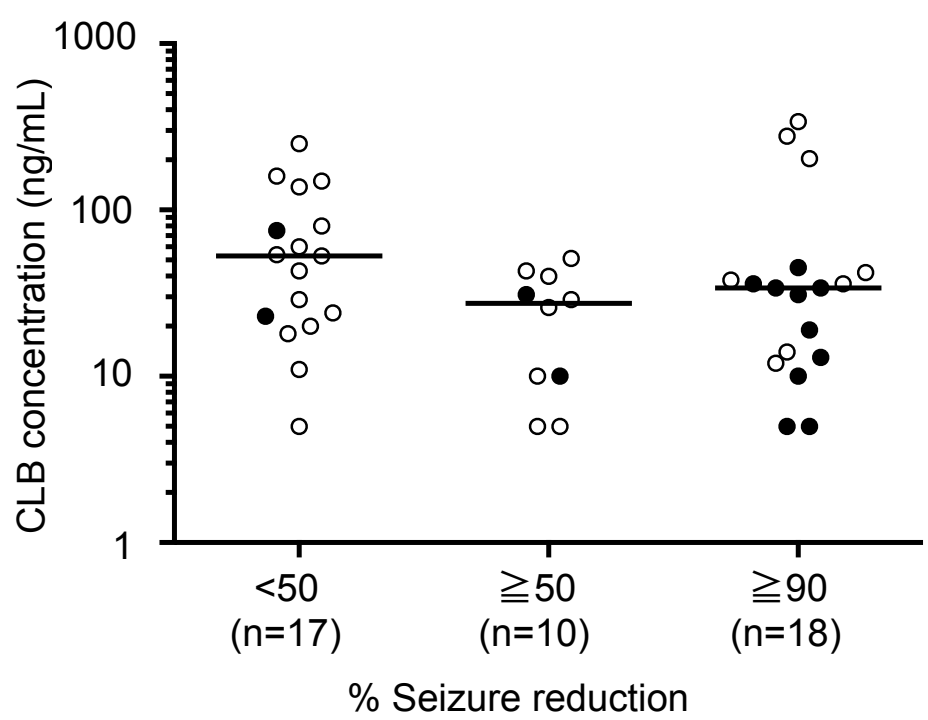

b)

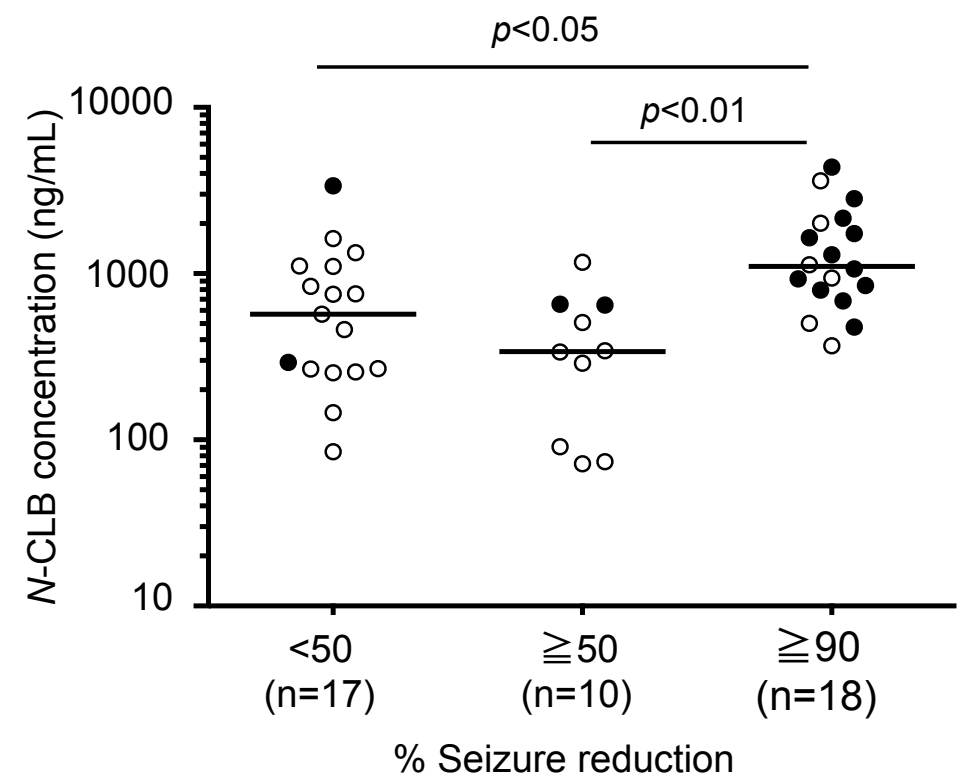

